\title{
Corona Virus Detection and Classification with radiograph images using $\mathrm{RNN}$
}

\author{
Dr Moulana Mohammed,Adapa V Krishna Raghavendra,Garapati Khyathi \\ Lahari \\ Bachelor of Technology in Computer Science and \\ EngineeringKoneru Lakshmaiah Educational Foundation \\ Deemed to be \\ UniversityGuntur, \\ AndhraPradesh
}

\begin{abstract}
We're working on detecting the symptoms of Corona virus, also known as Covid-19, in this project.COVID19 is a highly infectious disease that has been declared a Pub-lic Health Emergency and a Pandemic by the World Health Organization.The virus has infected over 25 million people worldwide, which has killed over 840,000 people and threat-ened the lives of millions more. COVID-19 is characterised by a dry cough, sore throat, and a high temperature. It is critical to find quick and accurate results for Covid-19 at this time in order to stop it in its early stages and avoid it from being a problem. Deep learning concepts are being used to analyse and classify symptoms from radiograph im-ages.Chest radiographs are one of the early screening tests to assess the onset of disease since the infection seriously affects the lungs.In this proposal, we used a recurrent neu- ral network model combined with a multi-level thresholding technique to detect Corona virus. One of the machine learn- ing techniques for prediction is the RNN model. A Recurrent Neural Network is used to decide if the given images belong to Covid-19 during the classification process. This implementation is based on a publicly available dataset of radiograph images.
\end{abstract}

Keywords:Covid-19 Detection Corona Virus, RNN, Ra- diographic images, Machine Learning.

\section{Introduction}

The COVID-19 coronavirus, also known as SARSCoV-2, has triggered a global crisis this will continue until the end of the year. COVID-19's cumulative incidence is steadily in- creasing day by day. Cloud computing and Machine Learn- ing (ML) can be very useful in monitoring the outbreak, fore- casting its progress, and modelling strategies and policies to stop it from spreading. This study employs a more advanced mathematical model to assess and forecast the epi- demic's progression. An improved ML-based model was used to forecast the potential hazard of COVID-19 in coun- tries around the world.We show that fitting the Generalized Inverse Weibull distribution with iterative weighting yields a better fit for creating a prediction system. On a cloud com- puting platform, this has been applied with a more precise and real-time forecast of the epidemic's spread. A data- driven strategy with greater precision can be quite usefulfor a constructive response from the government and people. Finally, we recommend a collection of research oppor- tunities and setting-up grounds for more practical applica- tions. For this paper, we get inputs from the image dataset.To make a prediction, we must first figure out which algorithm can effectively classify a given image as favourable or un- favourable. Reccurent Neural Networks were used to iden- tify them in this paper.

\section{Literature Survey}

Several studies in the literature have used X-ray data to demonstrate reasonable performance using various deep learning techniques[1]. DarkCovidNet, a model for early detection of COVID-19 that used 17 convolutional lay- ers to perform binary and multi-class classification involv- ing normal, COVID, and pneumonia cases, was proposed in. 
COVID-19 features in radiograph data can be detected and monitored using existing deep learning models on CT scan images[1]. Using CNN architectures like VGG-16 to distinguish between COVID-19 and non-COVID-19 cases[1].Deep learning was shown to be a viable technique for identifying COVID-19 from radiograph images in their experiments[1]. The trained approach is designed to distin- guish COVID-19 cases from normal cases as well as patho- logical patients with similar symptoms from other respira- tory disorders, such as pneumonia. For this reason, chest X-ray images from normal patients are grouped along with those from pathological patients with respiratory disordersother than COVID-19, and the method predicts Nor- mal andCOVID-19 groups. [2] In addition, we altered a network ar-chitecture to test the separability of the two types of chest X-ray images used in this study. To do this, we used a series of chest X-ray images from pa- tients infected withCOVID- 
19, patients with other patholo- gies with similar character-istics to COVID-19, and healthy patients to train a model.

[2] Several researchers have been working on the virus since it first spread, developing a variety of methods for detect-ing and dealing with Covid-19. The use of $\mathrm{x}$-rays in covid-19 prediction was inspired by the initial approaches used inpneumonia detection from chest $\mathrm{x}$ rays using deep learning models [3]. It's also vital to provide a good dataset with confirmed covid19 patients' chest X-ray images. (3). Transfer Learning is a method in which we train a modelfor one problem and then use it for a few similar problemswith mi- nor changes. In the new model, one or more layersfrom the learned model are used. [3,4] Instead of clinical results as- sociated with various conditions, a deep learning model will group X-ray images according to the scanning equip- ment used for the examination[2]. Furthermore, the vast majority of the photographs in these datasets were taken with fixed $\mathrm{X}$ - ray equipment. The capture equipment and acquisition tech- nique affect the quality of chest X-ray images in terms of spatial resolution, contrast, presence of objects, and noise[2]. Furthermore, as RNN and other automated feature extraction techniques have become more popular, interest in early-stage feature extraction techniques has begun to wane. The RecurrentNeuralNetwork architecture is a deep learning architec- ture that automatically extracts and categorises images from images. A hybrid model called fused perceptual hashbased RNNwas proposed to reduce the time it takes to classify CTimages of the liver while maintaining accuracy. To solve the problem of medical image unbalance, a transfer learning technique was used. Reference 19 used an optimal deep neu-ral network and linear discrimination analysis to analyse CT scans of lung images. Raw CT images were converted to low attenuation, and raw images and high attenuation pat- terns were rescaled. RNN was then used to re-sample and rate these three samples. Many researchers are working on this, beginning with the virus's result, and have discovered a num- ber of methods for detecting covid-19 and curing it. The useof $\mathrm{x}$-rays in covid-19 prediction was inspired by the initial approaches used in pneumonia prediction from chest X-raysusing deep neural networks[3]. Instead of clinical results associated with various conditions, a deep learning model will group X-ray images according to the scanning equip-ment used for the examination[2]. Furthermore, the vast ma-jority of the photographs in these datasets were taken withfixed Xray equipment. The capture equipment and acquisi-tion technique affect the quality of chest X-ray imageswhen it comes to spatial resolution, contrast, presence of objects, and noise[2]. Transfer Learning is a technique in which wedevelop a model for one problem and then apply it to a few similar problems with slight modifications[3]. In the newmodel, one or more layers from the learned model are used.It reduces the amount of time it takes to train a neural net- work for hyperparameter tuning. When we make use of pretrained transfer learning models, we typically freeze just a few or none of the layers of the model. VGG (VGG 16 or 19)[3] and VGG (VGG 16 or 19) are two common transfer learning methodologies. The current infrastructure for de- tecting COVID-19 positive patients (e.g. small image data sources with expert labelled data set) is inadequate, and manual detection takes a long time[4]. With the increase in global incidences, it is expected that a Deep learning-based solution will be developed and combined with clinical prac- tises to provide cost-effective, dependable, and simple auto- mated COVID-19 detection to aid the screening process[4]. X-ray scans are widely used by radiol- ogists to diagnose lung inflammation, swollen lymph nodes, and pneumonia. The COVID-19 virus infects the endothelial cells that line the lungs once within the body. X-rays may be used to deter- mine a patient's lung health. X-ray analy- sis requires an expert and takes a long time[4,3]. Hybrid approaches to merg-ing CNN and other ML algorithms are gaining popularity in the literature after outperforming cur- rent state-of-the-art in a variety of cases. Xiao-XiaoNiuetal. Created a Hybrid Algorithm that combined CNN and SVM to achieve a 95 percent digit recognition accuracy [5]. Ben Athiwaratkune- tal. in- troduced various hybrid algorithms in his paper and demon- strated that they worked better [5]. By removing a multi- perceptron layer from CNN, Shaoqing Ren et al. used Faster R$\mathrm{CNN}$ for feature extraction, and features were transferred to Random Forest, which performed better than CNN[5]. Various methods were used to analyse the chest X- rays, but the majority of them aren't very useful. Machine learning was used to help improve the 
algorithms, but the systems' accuracy was poor $[5,6]$. Deep learning and the Convolution Neural Network (CNN) began to have an im- pact in 2007 and things began to change [5]. Deep learn- ing algorithms have an inherent weakness in that they need a large data set to train[5].One of the most widely used tech-niques for diagnosing pneumo- nia is chest radiography (X- ray) [6]. A chest X-ray is a simple, low- cost, and widely used clinical procedure [6]. In contrast to computed tomog- raphy (CT) and magnetic resonance imag- ing (MRI), a chest X-ray exposes the patient to less radiation [2,6]. Making the right diagnosis from X-ray pictures, on the other hand, necessitates expert expertise and experi- ence $[5,6]$. A chest X-ray is much more difficult to diagnose than other imaging modalities like $\mathrm{CT}$ or MRI. COVID-19 can only be detected using a chest Xray by a specialized physician. There are fewer experts who can make this diagno- sis than there are general practitioners. In many countries around the world, even in normal times, the number of doc- tors per personis inadequate. According to 2017 statistics, Greece has the most doctors per 100,000 people, with 607 doctors. This figure is much smaller in other countries [6].The health sys- tem will collapse in the case of a disaster, including the COVID-19 pandemic, that necessitates simultaneous emer- gency care due to a lack of hospital beds and medical per- sonnel. COVID-19 is also a highly infectious disease, with physicians, nurses, and caregivers being especially vulnera- ble. Early diagnosis of pneumonia is critical for both slow- ing the spread of the virus and ensuring the patient's recovery [6]. Computer- aided diagnosis (CAD) helps doctors to diagnose pneumo- nia from a chest X-ray more rapidly and re-liably [6]. Artifi- cial intelligence approaches are becoming more common in the medical field because of their ability to work with large datasets that surpass human capacity [6].In-

tegrating $\mathrm{CAD}$ methods into radiologist diagnostic systems decreases doc- tors' workload while also enhancing accuracy and quantita- tive analysis [6]. Several deep learning-based methods for classifying lung diseases have been proposed and evaluated to be effective at the human level [7]. Almost all of these techniques, on the other hand, are designed to diagnose

\section{Experiments}

Neural Network par- ticular diseases like pneumonia [5,7], tubercu-losis [7], and lung cancer [7]. Meanwhile, researchers are working on a unified deep learning system for accurately de-tecting several common thoracic diseases [7]. Reverse tran- scription poly- merase chain reaction[8] confirms the diag- nosis of COVID- 19. The importance of chest radiography (CXR) is still a hot topic of debate. Present CXR studies on COVID-19 in- clude a range of terms as well as differentevaluations of its sensitivity and specificity. This can lead to CXR results be- ing misinterpreted, rendering comparisons between exami- nations and study studies difficult $[8,6]$. We recommend ter- minology for consistent CXR reporting andseverity assess- ment of individuals under investigation for COVID-19, pa- tients with a verified diagnosis of COVID- 19, and patients who may have radiographic symptoms of COVID-19, in or- der to satisfy this need for accuracy. When the diagnosis of COVID-19 is not suspected clinically, re- sults characteristic or indicative of COVID-19 are found[8]. The most common imaging test in the country is chest ra- diography, which is essential for screening, diagnosing, and treating a variety of life-threatening diseases [9]. From en- hanced workflow prioriti- sation and clinical decision sup- port to large-scale screening and global population health initiatives, automated chest ra- diograph interpretation at the level of practising radiologists could provide significant ben- efit in a number of medical set- tings[9]. The most popular image analysis requested is chest X-rays (CXRs).CXR Com-puterAided Diagnosis (CAD) has gotten a lot of attention in the scientific community, both be- fore and after the suc- cess of deep learning [10]. A recent effort has been made to build a new generation of CAD systems for the identification and visualization of common thoracic diseases from CXR images using advances in machine learning, especially deeplearning $[11,10,8]$.The area of time series forecasting knownas spatial-temporal time series is concerned with variables that shift over time and space. Disease forecasting, such as COVID19, would benefit from spatial-temporalforecasting because it analyses patterns and provides a reliable predic- tor for decisionmakers all over the world, ability to make theright decisions at the right time[12].

A Neural Network is made up of layers that are 
linked to operate on the structure and function of the human brain.It Contains an input layer, one or more hidden layers, and an output layer make up a neural network. One or more func- tion variables (or input variables or independent variables) denoted as $\mathrm{x} 1$, $\mathrm{x} 2, \ldots, \mathrm{xn}$ make up the input sheet. One or more secret nodes or hidden units make up the hidden layer. In the diagram above, a node is actually one of the circles.

The output variable, too, is made up of one or more outputunits

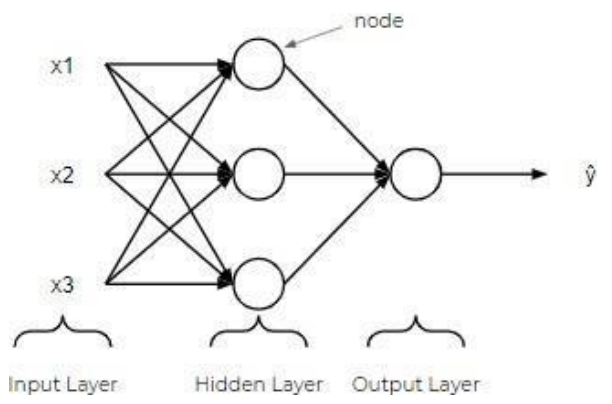

Fig. 1: simple neural network

\section{Popular Neural Networks}

- Feed-Forward Neural Network: Regression and classifica-tion problems in general.

- Convolutional Neural Network: Used to detect objects and classify images.

- Deep Belief Network: In the healthcare industry, it's usedto detect cancer.

- Recurrent Neural Network: Speech recognition,
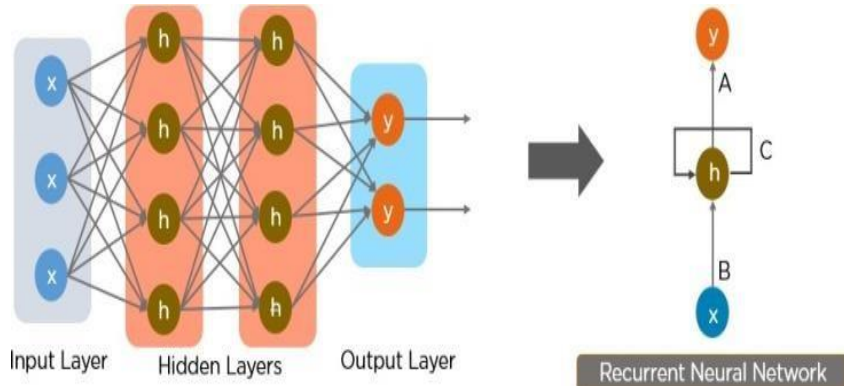

Fig. 2: Simple Recurrent Neural Network

\section{Applications of Recurrent Neural Networks}

- Time Series Prediction: An RNN can be used to solve any time series problem, such as forecasting voice recognition, time series prediction, and natural language pro-cessing are all possible applications.

\section{Recurrent Neural Network}

The output from the previous step is used as input in the cur-rent step in a rnn. stock prices in aspe- cific month.

- Natural Language Processing: An RNN for Natural Lan- guage Processing can be used to perform text mining and sentiment analysis (NLP). 
- Machine Translation: If the input is in a single language, RNNs can be used to translate the input into multiple lan- guages as output.

\section{Advantages of Recurrent Neural Network}

- An RNN remembers every piece of knowledge over time. It is only useful in time series prediction because it has the ability to remember previous inputs. This is referred to as Long Short Term Memory (LSTM).

- Even convolutional layers are used with recurrent neuralnetworks to extend the effective pixel neighbourhood.

\section{Disadvantages of Recurrent Neural Network}

- Problems of gradient disappearing and bursting.

- It is extremely difficult to train an RNN.

- When using tanh or relu as an activation feature, it won'tbe able to process very long sequences.

\section{Long Short Term Memory}

LSTM (Long Short Term Memory) networks are a form ofRNN that can learn long-term dependencies and are com- monly referred to as "LSTM." LSTM was developed with the goal of preventing long-term dependency. It is their in- nate ability to recall information for long periods of time. LSTM retains the error that can be back-propagated across time and layers. They allow repeated networks to continue to learn by sustaining a more constant error over a large num-ber of stages (over 1000), thus opening up a channel for re- mote connections between causes and consequences. LSTMstores data in a gated cell outside of the recurrent network'snormal flow.A cell's contents can be saved, written, or read.The neuron makes decisions about what to store and makes it possible to read and write by opening and closing gates. The layers are as follows:

1. Tokenization: This isn't an LSTM network layer, but rather a necessary transformation of our phrases into tokens (integers).

2. Embedding Layer: Transforms our term tokens (integers)to the required size for embedding.

3. LSTM Layer (Long Short Term Memory): Unknown stateand number of layers discovered.

4. Completely Connected Layer: Mapping the output of the LSTM layer to the output size desired.

5. Sigmoid Activation Layer: In this layer, all output values will be translated to values ranging between 0 and 1.

6. Output: The final network's development is considered the final time stage of Sigmoid production.

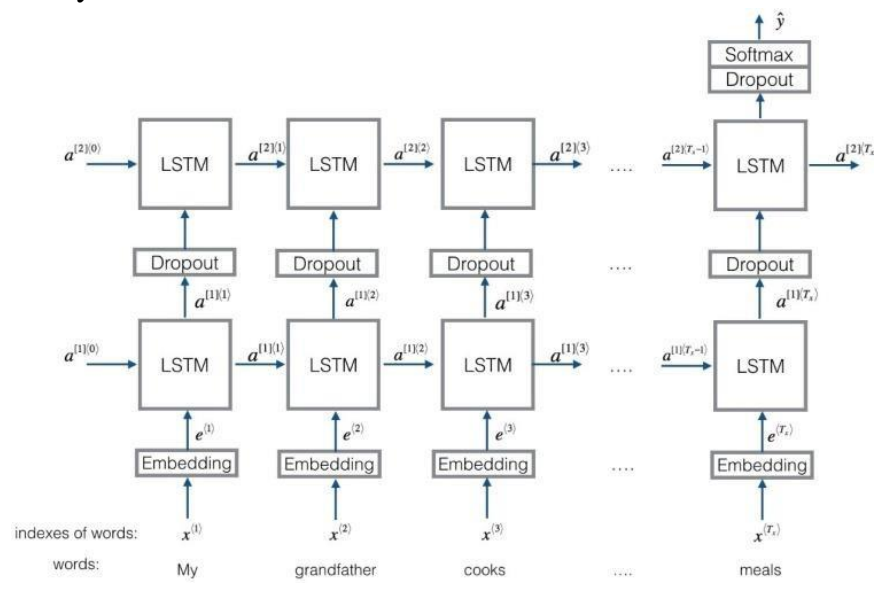

Fig. 3: Long-short term memory architecture

\section{Memory Cell}

This is a specialised neuron that is responsible for recognis- ing long-term dependencies. The LSTM employs a vector that moves from one cell to the next in its inner state. The LSTM is smart enough to figure out just how long it would take to recall old data and skip the step of figuring out how to link new information to old memories. 


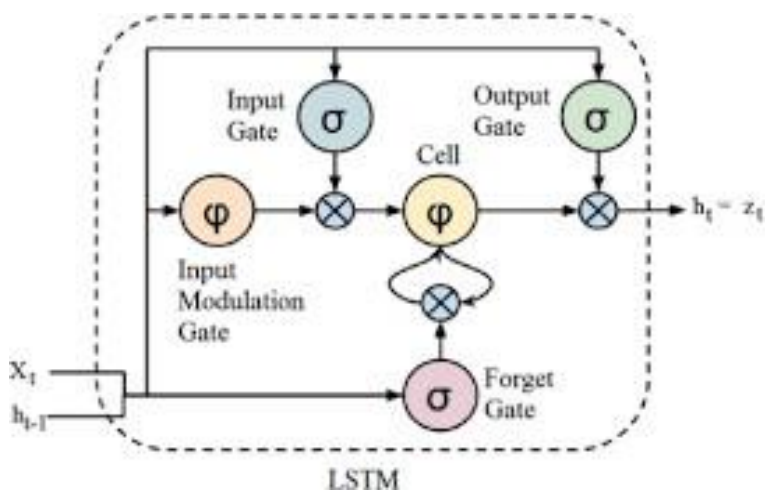

Fig. 4: Architecture of memory cell in LSTM

We'll construct an LSTM model using word normal healthy conditions. sequences as data, with word ordering taken into account. To define terms, we'll use GloVe's 50dimensional pre-trained word integra-tions. Then, as an input, we feed the word em-beddings into an LSTM, which predicts the most appropriate emotion for the text. Hidden states are output by the LSTM, which takesembedded words as input.The linear layer that transforms hidden state space into output space.

\section{Applications of LSTM}

- Robot control: Robust control is a controller design tech- nique that directly addresses ambiguity. Robust control methods are designed to work properly when there are un- known parameters or disturbances in a given collection.

- Speech recognition: Speech recognition, also known as au-tomatic speech recognition (ASR), machine speech recogni- tion, or speech-to-text, is a feature that allows a computer programme to translate human speech into text.

- Handwriting recognition: Handwriting recognition (HTR) is the ability of a computer to obtain and interpret intelligi- ble handwritten information from sources such as paper doc- uments, images, touch screens, and other devices..

\section{Proposed Methodology}

In order to predict the Covid-19 disease, we implemented a few of the Artificial Neural Network concepts RNN in this paper.The traditional method of gathering the dataset (col- lection of CSV datasets) is delegated by collecting the im- age datasets. To be more specific, the dataset contains radio- graphic images of patients with pneumonia, Covid-19, and 


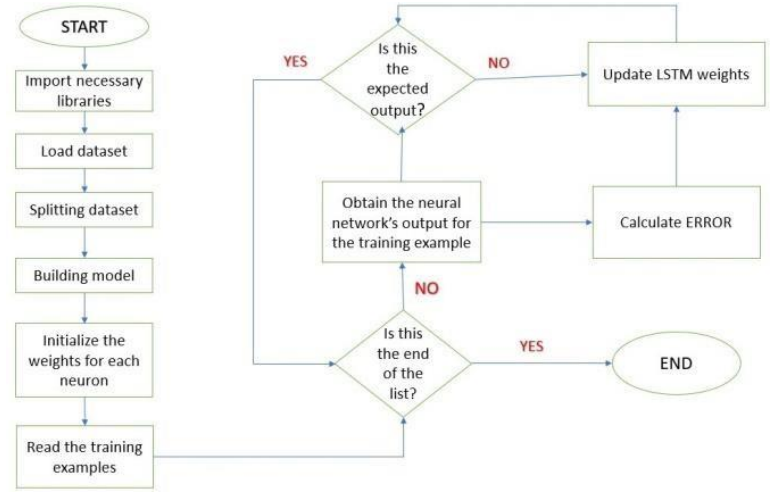

Fig. 5: FlowChart for implementation

Keras is used inorder to perform the image classification for the dataset, these three types of images, as well as their names, are all translated to bytes and integers. and these are stored into XML file and are further slip into training and testing.The data contains the images of three categories: Normal, Covid and Viral pneumonia.These data files are extracted into a list and converted as training and testing model. After converstion the data is stored in the $\mathrm{xml}$ file as bytes and integers. The xml file is used for comparing the Covid images and Viral pneumonia images with the Normal ones and gives the accuracy of barely 0.7.As comapared to CNN model, RNN model shows a bit low performance, so this model shouldn't be considered for image classification. than, training loss in most cases. Continue doing more trainingas long as the loss of validation is less than or even equal to the loss of training. If your training loss decreases but your validation loss does not, you should increase your train-ing. When the lack of validity starts to rise, it's time to stop. With radiographic images, we got an accuracy of 86 percent, 93 percent with CT-Scan images, and 76 per- cent with $\mathrm{X}$-Ray images. We conclude that using CT-Scan images for Covid-19 classification is better than using Ra- diographic images and X-Ray images after comparing the results of Radiographic images, CTScan images, and X-Rayimages. The RNN model performs poorly as compared to the CNN model, so it should not be used for image classifi- cation.

\section{Experimental Results}

Validation loss should be similar to, but slightly greater

\section{Results of Radiographic Images}

\begin{tabular}{l|l|l|l|l} 
Epoch & Loss & Accuracy & Val_Loss & Val_Accuracy \\
\hline 1 & 0.7313 & 0.6162 & 0.6861 & 0.6901 \\
\hline 2 & 0.6225 & 0.6802 & 0.5742 & 0.7423 \\
\hline 3 & 0.5860 & 0.7223 & 0.5106 & 0.7756 \\
\hline 4 & 0.4788 & 0.7967 & 0.4541 & 0.8069 \\
\hline 5 & 0.4401 & 0.8163 & 0.3749 & 0.8310 \\
\hline 6 & 0.4208 & 0.8275 & 0.3853 & 0.8345 \\
\hline 7 & 0.4008 & 0.8398 & 0.3475 & 0.8485 \\
\hline 8 & 0.3697 & 0.8575 & 0.3241 & 0.8620 \\
\hline 9 & 0.3607 & 0.8597 & 0.3175 & 0.8832 \\
\hline 10 & 0.3638 & 0.8604 & 0.3088 & 0.9201
\end{tabular}

Fig. 6: Accuracy of Radiographic Images

$\begin{array}{rrrrr} & \text { precision } & \text { recall } & \text { fl-score } & \text { support } \\ \text { COVID } & 0.92 & 0.84 & 0.91 & 812 \\ \text { NONCOVID } & 0.75 & 0.83 & 0.78 & 575 \\ & & & & \\ \text { accuracy } & & & 0.86 & 1387 \\ \text { macro avg } & 0.86 & 0.87 & 0.86 & 1387 \\ \text { weighted avg } & 0.87 & 0.86 & 0.86 & 1387\end{array}$


Fig. 7: Model Results

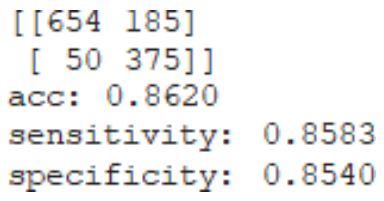

Fig. 8: Confusion Matrix
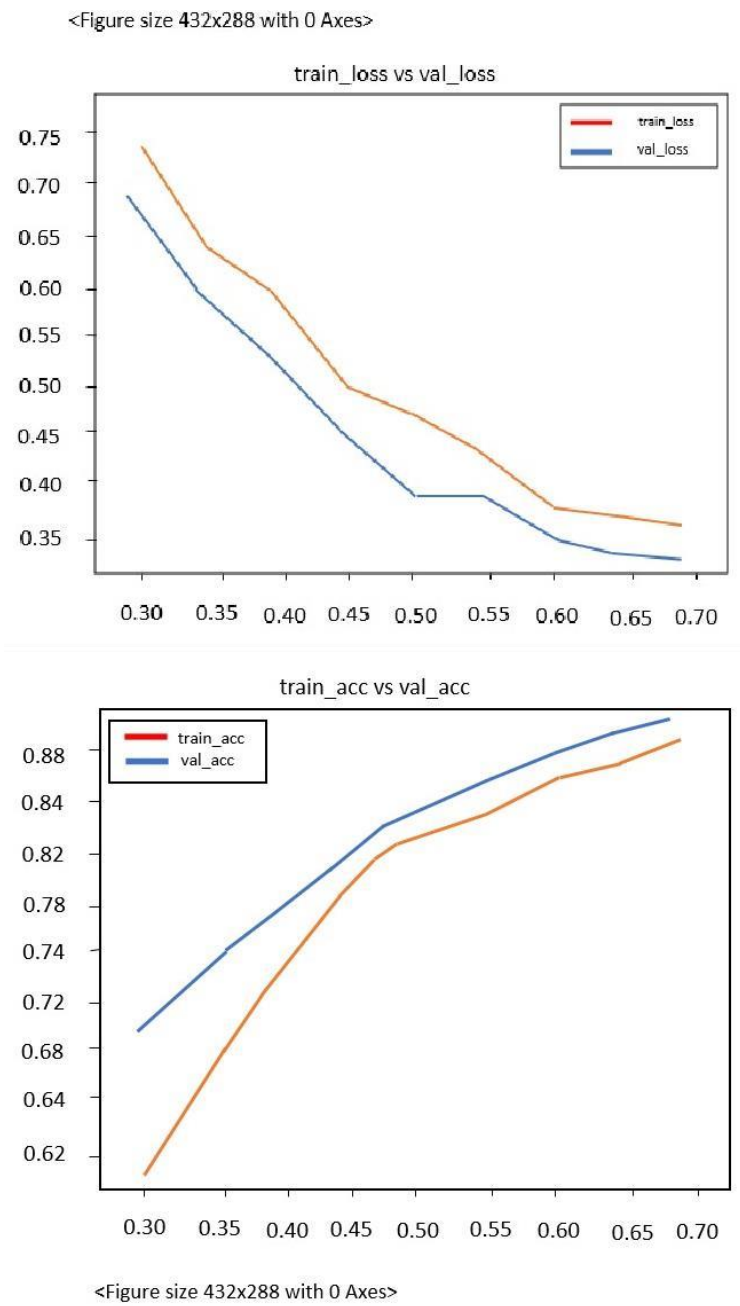

Fig. 9: Model graph 


\section{Results of CT-Scan images}

\begin{tabular}{l|l|l|l|l} 
Epoch & Loss & Accuracy & Val_Loss & Val_Accuracy \\
\hline 1 & 0.4469 & 0.7720 & 0.3117 & 0.8371 \\
\hline 2 & 0.2771 & 0.8691 & 0.2290 & 0.8925 \\
\hline 3 & 0.2328 & 0.8904 & 0.2076 & 0.9090 \\
\hline 4 & 0.1958 & 0.9138 & 0.1906 & 0.9239 \\
\hline 5 & 0.1736 & 0.9223 & 0.1714 & 0.9256 \\
\hline 6 & 0.1492 & 0.9397 & 0.1629 & 0.9305 \\
\hline 7 & 0.1407 & 0.9410 & 0.1660 & 0.9280
\end{tabular}

Fig. 10: Accuracy of ct scan

$\begin{array}{rrrrr} & \text { precision } & \text { recall } & \text { fl-score } & \text { support } \\ \text { COVID } & 0.96 & 0.93 & 0.95 & 834 \\ \text { NONCOVID } & 0.86 & 0.92 & 0.89 & 375 \\ \text { accuracy } & & & & \\ \text { macro avg } & 0.91 & 0.93 & 0.93 & 1209 \\ \text { weighted avg } & 0.93 & 0.93 & 0.93 & 1209\end{array}$

Fig. 11: Model Results

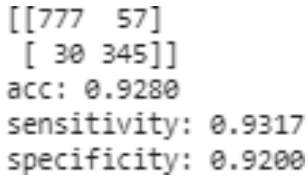

Fig. 12: Confusion Matrix
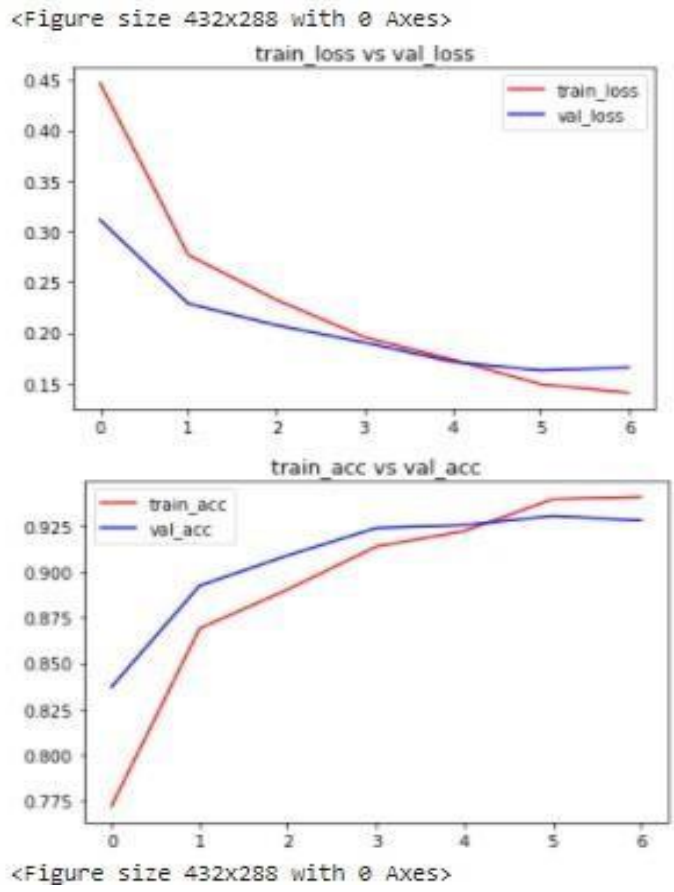

777 
Fig. 13: Model graph 


\section{Results of X-Ray images}

\begin{tabular}{l|l|l|l|l} 
Epoch & Loss & Accuracy & Val_Loss & Val_Accuracy \\
\hline 1 & 0.6300 & 0.6494 & 0.5912 & 0.7051 \\
\hline 2 & 0.5547 & 0.7249 & 0.5407 & 0.7331 \\
\hline 3 & 0.5276 & 0.7432 & 0.5148 & 0.7510 \\
\hline 4 & 0.5087 & 0.7580 & 0.5081 & 0.7617 \\
\hline 5 & 0.4879 & 0.7634 & 0.4973 & 0.7638
\end{tabular}

Fig. 14: Accuracy of X-Ray scans

$\begin{array}{rrrrr} & \text { precision } & \text { recall } & \text { fl-score } & \text { support } \\ \text { COVID } & 0.82 & 0.74 & 0.78 & 803 \\ \text { NONCOVID } & 0.70 & 0.80 & 0.75 & 628 \\ \text { accuracy } & & & 0.76 & 1431 \\ \text { macro avg } & 0.76 & 0.77 & 0.76 & 1431 \\ \text { weighted avg } & 0.77 & 0.76 & 0.76 & 1431\end{array}$

Fig. 15: Model Results

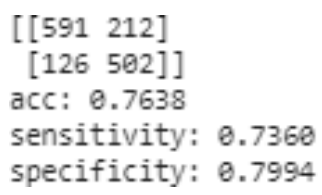

Fig. 16: Confusion Matrix
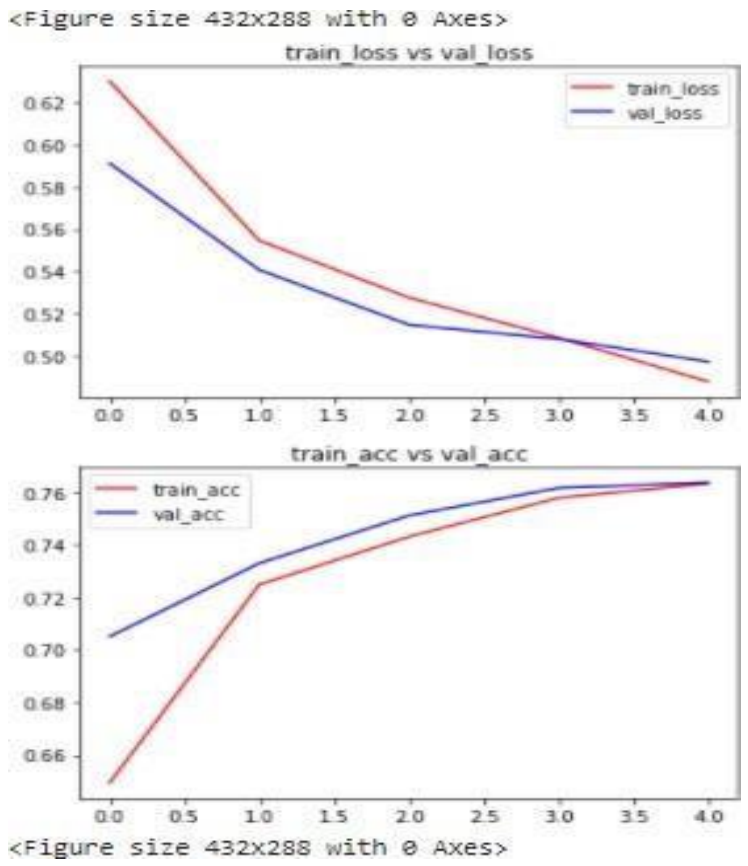

Fig. 17: Model graph

\section{Conclusion}

Covid-19 can be predicted in a variety of ways, 779 
including cough, cold, fever, tastelessness, and so on. Many of these things belong in the past. In this study, we discovered a new method for predicting the Covid19. Symptom inputs are usually obtained in the form of a CSV, which is a dataset whose results aren't entirely accurate. We've created a new form of data set that includes radiographic images. We have used machine learning concepts such as RNN, Lstm's. According to our results, rnn has the lowest accuracy comparedto cnn.

\section{References}

[1] DL-CRC: Deep Learning-Based Chest Radiograph Classification for COVID-19 Detection: A Novel ApproachSakib, Sadman, TahratTazrin, Mostafa M. Fouda, Zubair Md Fadlullah, and Mohsen Guizani. "DL-CRC: Deep Learning- Based Chest Radiograph Classification for COVID-19 Detection: A Novel Approach." IEEE Access 8 (2020): 171575-171589.

[2] Deep Convolutional Approaches for the Analysis of COVID-19 Using Chest X-Ray Images From Portable Devices De Moura, Joaquim, Lucía Ramos García, Plácido Francisco Lizancos Vidal, Milena Cruz, Laura Abelairas López, Eva Castro Lopez, Jorge Novo, and Marcos Ortega. "Deep convolutional approaches for the analysis of covid-19 using chest $x$-ray images from portable devices." IEEE Access 8 (2020): 195594-195607.

[3] Covid Prediction from X-ray

[4] "Covid Prediction from X-ray Images." In 2020 5th Inter- national Conference on Computing, Communication and Security (ICCCS), pp. 1-5. IEEE, 2020.

[5] Estimating Uncertainty and Interpretability in Deep Learning for Coronavirus (COVID-19) Detection Ghoshal, Biraja, and Allan Tucker. "Estimating uncertainty and in- terpretability in deep learning for coronavirus (COVID-19) detection." arXiv preprint arXiv:2003.10769 (2020).

[6] Hybrid deep learning Approach to evaluate chest ray im- ages for the diagnosis of pneumonia in children Abbasa, Mo-hammad Ali, Syed Usama Khalid Bukhari, and Syed
Khuza-imaArssalanBokhari. "The application of Hybrid deep learn- ing Approach to evaluate chest ray images for the diagnosis of pneumonia in children." medRxiv (2020).

[7] Automatic Detection of Coronavirus Disease (COVID-19) Using X-ray Images and Deep Convolutional Neural Networks(https://arxiv.org/ftp/arxiv/papers/2003/20 03.10849.pdf) Narin, A., Kaya, C., Pamuk, Z. (2020). Automatic detec- tion of coronavirus disease (covid-19) using x-ray images and deep convolutional neural networks.arXiv preprint arXiv:2003.10849.

[8] Interpreting chest $\mathrm{X}$-rays via CNNs that exploit hi-erarchical disease dependencies and uncertainty labels (https://arxiv.org/pdf/1911.06475.pdf) :Pham, Hieu H., Tung T. Le, Dat Q. Tran, Dat T. Ngo, and Ha Q. Nguyen. "Interpreting chest X-rays via $\mathrm{CNNs}$ that exploit hierarchical disease dependencies and uncertainty labels." arXiv preprintarXiv:1911.06475 (2019).

[9] Review of Chest Radiograph Findings of COVID-19 Pneumonia and Suggested Reporting Language Lit- manovich, Diana E., Michael Chung, Rachael R. Kirkbride, Gregory Kicska, and Jeffrey P. Kanne. "Review of chest radiograph findings of COVID-19 pneumonia and suggested reporting language." Journal of thoracic imaging 35, no. 6 (2020): 354-360.

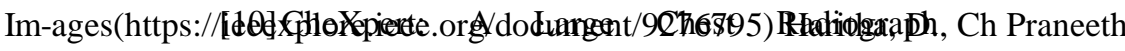
Dataset with Uncertainty Labels and ExpertComparison(https://arxiv.org/pdf/1901.07031v1.pdf) Irvin, Jeremy, et al. "Chexpert: A large chest radiograph dataset with uncertainty labels and expert comparison." Proceedings of the AAAI Conference on Artificial Intelligence. Vol. 33. No. 01. 2019.

[11] Deep Hierarchical Multi-labelClassification of Chest X-ray Images (http:/ /proceedings. mlr.press/ v102/chen19a/ chen19a.pdf) Chen, Haomin, Shun Miao, Daguang Xu, Gregory D. Hager, and Adam P. Harrison.'Deep hierarchical multi-label classification of chest X-ray images.” In International Conference on 
Medical Imaging with Deep Learning, pp. 109-120. PMLR, 2019.

[12] Interpreting chest $\mathrm{X}$-rays via CNNs that ex- ploit disease dependencies and uncertainty labels (https://arxiv.org/pdf/1911.06475v1.pdf)

[13] Pham, Hieu H., Tung T. Le, Dat Q. Tran, Dat T. Ngo, and Ha Q. Nguyen. "Interpreting chest $\mathrm{X}$-rays via CNNs that exploit hierarchical disease dependencies and uncertainty labels." arXiv preprintarXiv:1911.06475 (2019).

[14] Machine Learning Approaches for COVID-19 Forecast-ing Istaiteh, Othman, et al. "Machine Learning Approaches for COVID-19 Forecasting." 2020 International Conferenceon Intelligent Data Science Technologies and Applications (IDSTA). IEEE, 2020. 